\title{
ESTIMATION OF ENGAGEMENT CONDITIONS USING AN ANN PATTERN RECOGNITION SYSTEM ON THE BASE OF A SENSORY TOOL HOLDER
}

\author{
T. Bergs ${ }^{1}$, D. Schraknepper ${ }^{1}$, S. Goetz ${ }^{1}$ * \\ ${ }^{1}$ RWTH Aachen University, Laboratory for Machine Tools and Production Engineering, Aachen, Germany \\ *Corresponding author; e-mail: s.goetz@wzl.rwth-aachen.de
}

\begin{abstract}
Knowledge of the tool wear state in machining has become an important issue in research and industrial application. Current systems use the spindle power or cutting force as measured variable and refer it to a taught set point. However, this method lacks the ability to adapt to new work piece geometries. This new approach focusses on the tool instead of the workpiece, and uses a sensory tool holder with integrated strain gauges. Process monitoring that is based on the increasing effect of tool wear on the cutting force requires online information of the current engagement conditions to normalize the force information. The research shown in this paper hence presents a method to estimate the width of cut in end milling. The signal of the bending torque of the tool holder in the XY-plane shows a characteristic shape for each respective engagement condition. An analytically describable relationship has been found between these shapes for different engagement conditions. On the base of this relationship, an ANN was taught to estimate the width of cut without prior knowledge of the cutting path. The results show an accurate estimation for a wide range of cutting parameters.
\end{abstract}

\section{Keywords:}

Process monitoring; milling; Industry 4.0; ANN

\section{INTRODUCTION}

\subsection{Problem statement}

The tool wear state is an important information in manufacturing processes. It defines the tool costs and is a measure for the threat of fatal tool failure, often resulting in harm to the work piece, the tool holder or the machine. Excessive tool wear can lead to geometric errors of the work piece if the cutting edges are eroded. Hence, companies aim to monitor the tool wear state. Current state of the art are envelope-based systems. These systems need to be taught, and any change in the process renders the defined envelope obsolete. This calls for systems that monitor the tool instead of the process. Those systems would extract features from the measurement signal (e.g. power, force, temperature) to describe the tool wear state.

\subsection{State of the art}

Monitoring of tool wear can be distinguished between online and offline monitoring, where the offline monitoring still prevails in industrial use due to a lack of reliable online tool wear monitoring systems [Teti 2010]. Recent research focused on developing online methods for tool wear monitoring, and some systems are already commercially available. These systems usually rely on indirect measurement, such as cutting forces, vibration, acoustic emission, spindle motor power and feed currents [Tet 2010]. Tool wear results in an increase of both the cutting force and the process power [Klocke 2016]. Industrial praxis is the teaching of set-curves from "good" processes (e.g new tools, no chatter etc.) and the later comparison of signals from a process to this set-curve [Artis 2011]. This method has the disadvantage of inflexibility towards changes of the process or work piece. Any such change requires a new teaching of a set-curve. Modern research therefore aimes at finding ways to develop monitoring systems that are independent on the work piece and thus need no set-curves [Fussel 2015; Salgado 2013]. NouRI ET AL. propose a method to track the coefficients of a forcemodel that are independent on the engagement coefficients. However, this method requires exact information about the current engagement condition a priori, as well as the phase angle of the spindle [NOURI 2015]. SALGADO ET AL. propose a similar method that uses cutting-condition independent coefficients. These coefficients base on the kurtosis of the force signal, showing strong correlation to the flank wear. However, this method is only validated by turning experiments, where the engagement conditions are stable throughout the process. [Salgado 2013] Other monitoring systems also rely on knowledge about the cutting path or the engagement conditions [Emec 2016; Jáuregi 2018]. There is currently no system available to detect the engagement conditions online, to support those systems.

\subsection{Research aim and approach}

As shown in Chapter 1.2, current state-of-the-art tool wear monitoring systems rely on the knowledge of the engagement conditions. Those can be computed, but this is often very time consuming. The research aim of this paper is hence to provide a new method to compute the engagement conditions from online measurement signals. This is based on the load torques of the tool holder for an end milling process. Since the reference coordinate system 
of such a measurement system is stationary with respect to the tool, it provides characteristic information about the current process state. This can be expressed in polar figures. Due to its spiral flutes, the interplay of the individual cutting edges of an end milling tool depends on the width and depth of cut, $a_{e}$ and $a_{p}$. It can be shown that the polar figures have characteristic features depending on the width of cut $a_{e}$. The aim of this research paper is to define a new method to extract these features in an online method, using an Artificial Neural Network (ANN) to find the width of cut from them. The outcome is validated with a series of machining experiments with different widths of cut.

\section{EXPERIMENTS AND DATA ANALYSIS}

This chapter describes the experiments conducted as basis for the current research. Chapter 2.1 explains the fundamentals of polar figures, 2.2 the experimental buildup. 2.3 analyzes the data and describes the method concept for the $a_{e}$ identification.

\subsection{Fundamentals of polar figures}

The cutting force, acting on the milling tool, causes a bending moment on the tool holder. This can be measured by piezo-electrical sensors or strain gauges. When these sensors are attached $90^{\circ}$ to each other, it is possible to measure the components of the resultant bending moment, $M_{x}$ and $M_{y}$. These measurements are stationary with respect to the tool. $M_{x}$ and $M_{y}$ hence give information about the load acting on the tool for any given time. The relation between $M_{x}$ and $M_{y}$ also carries information about the direction of this load. When they are interpreted as coordinates of a Cartesian coordinate system, one receives a pattern that is time invariant and displays characteristic features of the current load situation of the tool. This method is displayed in Figure 1. In the further paper, the result of this method is called "polar figure".

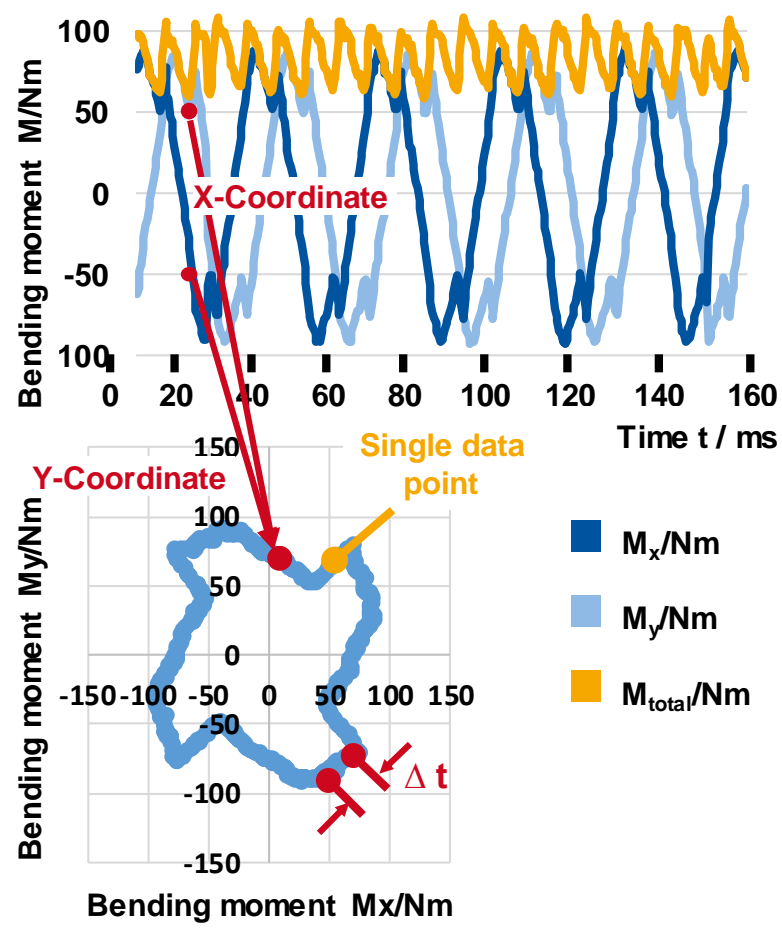

Fig. 1: Concept of polar figures.

The upper part of Figure 1 shows $\mathrm{M}_{\mathrm{x}}, \mathrm{M}_{\mathrm{y}}$ and their resultant $M_{\text {total }}$ in the time domain. The lower part shows the polar figure derived from these signals, where for each $t_{i}$ the respective $M_{x, i}$ and $M_{y, i}$ are interpreted as one point of the polar figure, which have a temporal distance of $\Delta t$. The sum of these points resolves into the polar figure over the course of one tool rotation. In a constant process, several consecutive tool rotations overlay each other with similar polar figures. In the likely case that the sample rate is no integral multiple of the rotational speed, the data points of any subsequent rotation are offset to the prior one. This leads to a higher "virtual" sample rate when more than one rotation is regarded, c.f. Figure 2.

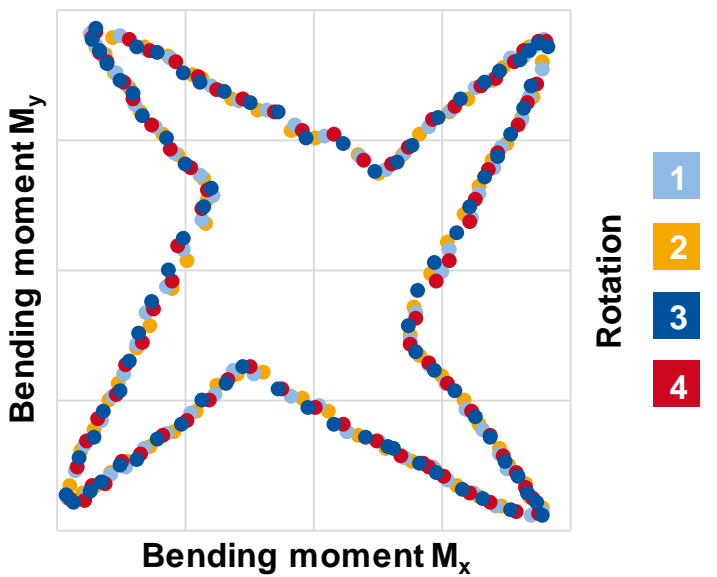

Fig. 2: Virtual sample rate for four tool rotations.

\subsection{Description of experimental setup}

The cutting experiments were conducted using an end mill with $z=4$ cutting edges, a diameter of $d=12 \mathrm{~mm}$ and a helix angle of $\lambda=28^{\circ}$. The specimen material was a standard construction steel C20 (1.0038). The process parameters were as follows: Cutting speed $v_{c}=75 \mathrm{~m} / \mathrm{min}$, the feed per tooth $f_{z}=0.03 \mathrm{~mm}$, the depth of cut $a_{p}=[5 ; 10 ; 15] \mathrm{mm}$ and the width of cut $a_{e}=[1 ; 2 ; 3 ; 4 ; 5] \mathrm{mm}$. This resulted in 15 experimental cuts, during all of which the bending moment on the tool holder was measured using a "SPIKE mobile" ${ }^{\circledR}$ measurement device. The sample rate was $f_{s}=2,500 \mathrm{~Hz}$. During the experiments, the end mill was changed in such a way that tool wear effects can be neglected.

\subsection{Analysis of the data and method concept}

The polar figures from all 15 experiments were analyzed with respect to characteristics that change with the engagement conditions. Most noticeable is a characteristic that changes with the width of cut $a_{e}$. Figure 3 shows the five polar figures for a depth of cut $a_{p}$ of $15 \mathrm{~mm}$. The figure shows four regions where the five polar figures form one common line of data points, marked in orange. This region shall be defined as "identification region". Each of these regions is formed from parts of the polar figures. Polar figures from processes with smaller $a_{e}$ contribute the parts closer to the origin and polar figures from processes with larger $a_{e}$ the parts further from it. This indicates a relationship between parts of the polar figures with their corresponding width of cut. The first point along the time flow of a polar figure (c.f. Figure 3 ) that lies within the identification region shall be defined as entrance point, the last point inside this region as exit point. These entrance and exit points are ranked according to their respective width of cut. For example, the polar figure of $a_{e}=2 \mathrm{~mm}$ enters the identification region at point $A$ and exits it at point B. For $a_{e}=5 \mathrm{~mm}$, this happens at points $C$ and $D$, respectively. $\mathrm{C}$ is considerably further from the origin than $A$, and it gets apparent that the entrance points for $a_{e}=3 \mathrm{~mm}$ and $4 \mathrm{~mm}$ lie in between $A$ and $C$ along the orange line. 


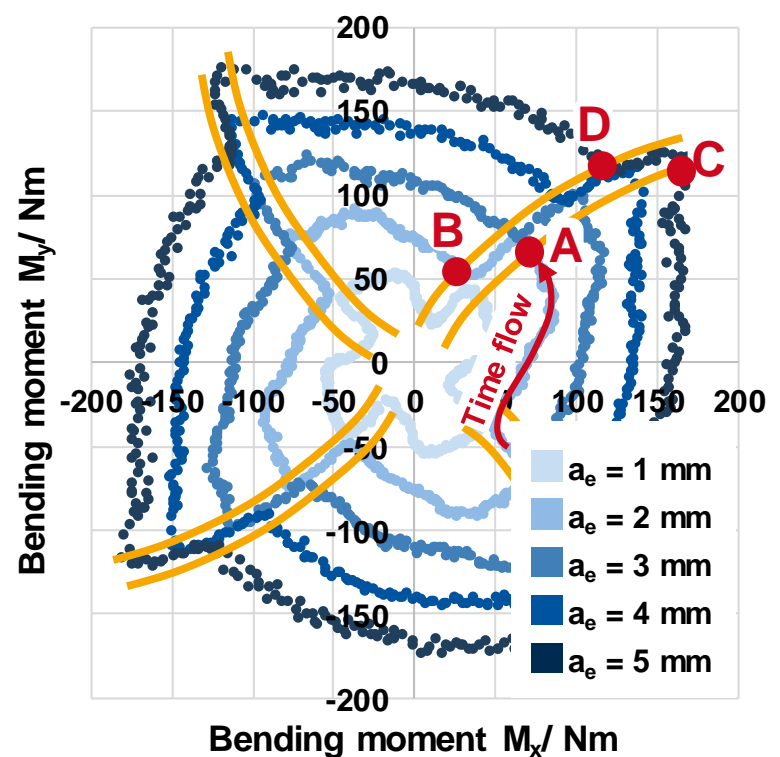

Fig. 3: Characteristic features of polar figures with different $a_{e}$ values.

This strongly indicates a systematic relationship between the width of cut and the entrance and exit points of a polar figure along a certain region that is defined by the mutual combination of polar figures with different widths of cut. This fact shall be used to develop a method to estimate the current width of cut from the polar figures.

Figure 3 shows that the identification region can be defined for a set of polar plots with the same depth of cut, but different widths of cut. It hence needs to be analyzed how the identification region is influenced by the depth of cut. Figure 4 shows exerts from 10 different polar plots with a depth of cut of $a_{p}=10$ and $15 \mathrm{~mm}$.

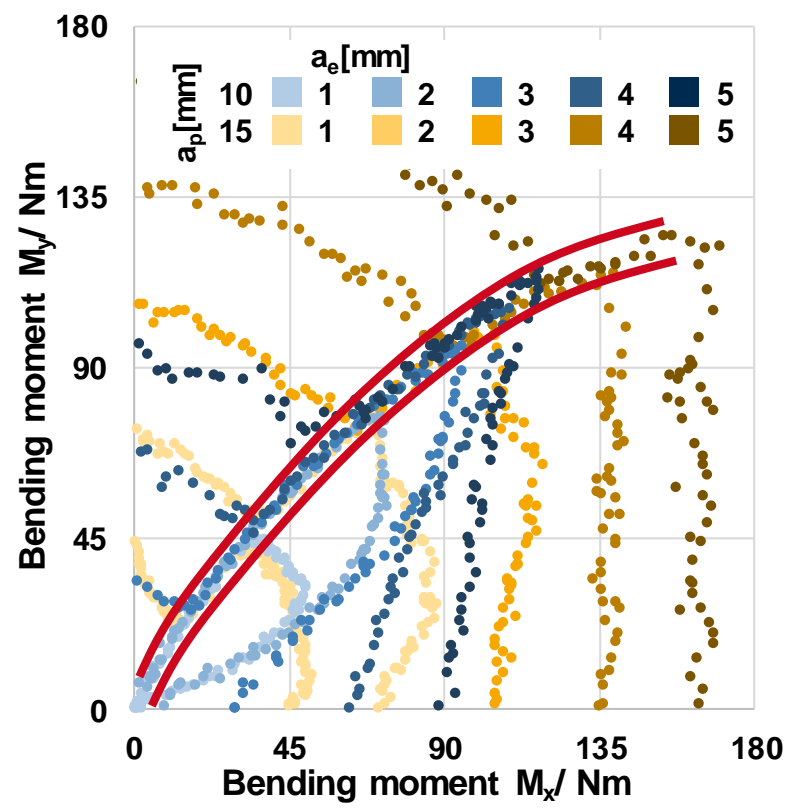

Fig. 4: Identification region for $a_{p}=10 \mathrm{~mm}$ and $15 \mathrm{~mm}$.

The identification regions from both $a_{p}$ groups coincide with each other. This indicates that the identification region does not depend on the process parameters, but solely on the geometry of the tool. However, the entrance and exit points of polar figures of a specific width of cut lie towards larger bending moments for larger depth of cuts. To prove this, a second set of experiments was conducted with different cutting speed $v_{c}$ and feed per tooth $f_{z}$. The aim was to show that the shape of a polar figure, and thus the definition of the identification region, is independent on these parameters. Figure 5 shows the polar figures of these experiments.

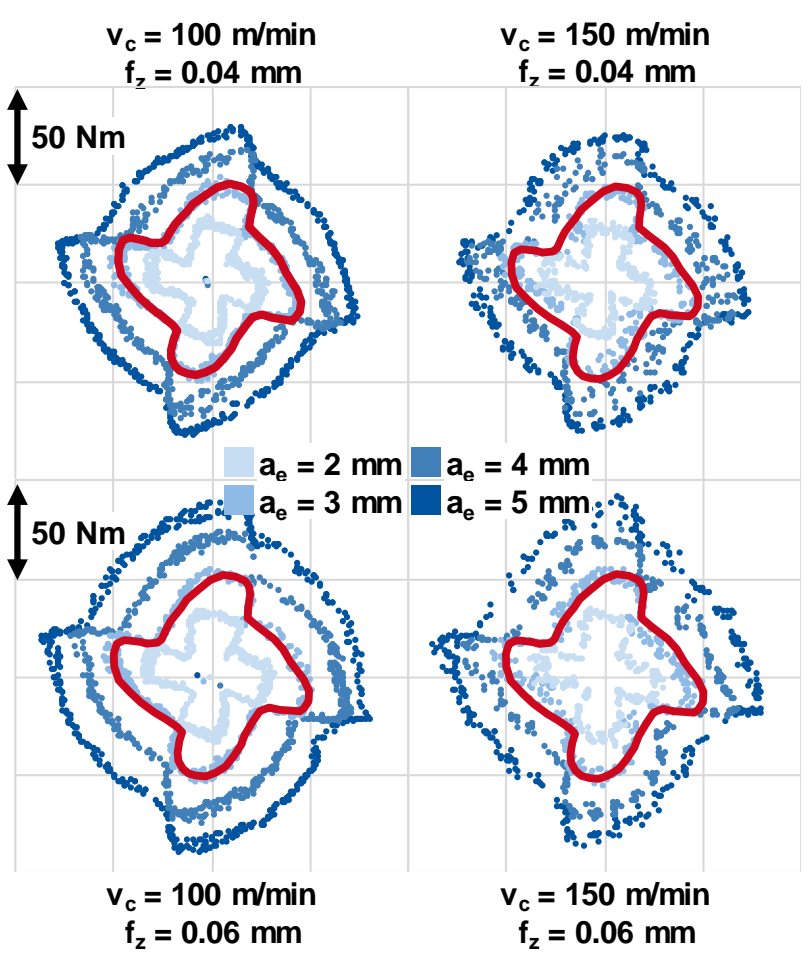

Fig. 5: Influence of cutting speed and feed rate on polar figures.

The red line indicates the course of the polar figure for $a_{e}=3 \mathrm{~mm}$. It is apparent that despite a change in size, the shape itself stays unchanged for different cutting speeds and feed rates. If the shape of a polar figure is unchanged, so is the resulting identification region.

A method to estimate the width of cut $a_{e}$ from polar figures is therefore robust against changes of the process parameters. The method concept consists of the definition of the identification region, the determination of the entrance and exit points and the interpretation of these points into values for the width of cut.

\section{METHOD DEVELOPMENT}

\subsection{Theoretical background}

The estimation method for the width of cut relies on the entrance and exit points into and from the previously defined identification region. In order to be able to quantify this, the identification region is subdivided into percentiles according to Figure 6 (left).

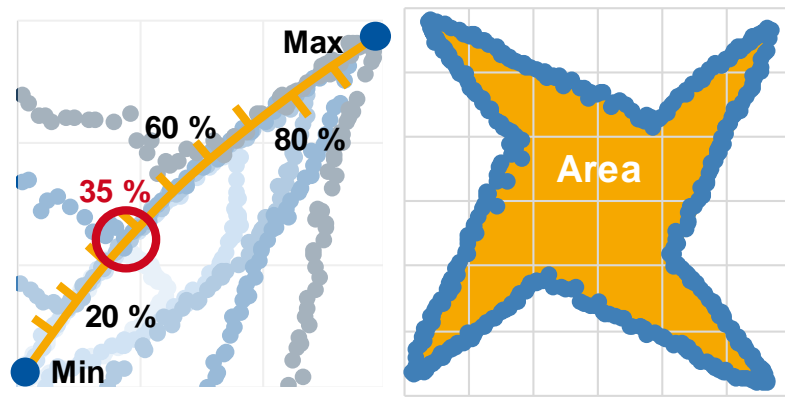

Fig. 6: Definition of percentage and area of polar figures. 
The minimum and maximum points of the region are used as reference for $0 \%$ and $100 \%$, respectively. Figure 6 (left) shows exerts of three polar figures with $a_{p}=10 \mathrm{~mm}$ and $a_{e}$ of 3,4 and $5 \mathrm{~mm}$. The polar figure of $a_{e}=4 \mathrm{~mm}$ exits the identification region at $35 \%$ of its maximum value (red circle). Likewise, percentages can be defined for all entrance and exit points. As can be seen in Figure 4, the entrance and exit points for the same width of cut but larger depth of cut are at a higher percentage (further away from the origin). This aspect needs to be considered in the development of the method.

A second quantity describing the engagement situation is the area enclosed by the polar figure. With increasing engagement, either by a larger depth or by a larger width of cut, the load on the tool increases. As the polar figure is representing the integral load on the tool over one tool rotation, its area correlates strongly with the tool load and thus with the engagement conditions. However, the area alone is not suitable for detecting the engagement conditions, as a change of it can be induced by both the width or the depth of cut.

The entrance and exit points as well as the area, the depth of cut and the quadrant are used as input variables for the method. This will be further described in the next chapter.

\subsection{Description of method}

The method to estimate the width of cut from the polar figures uses an Artificial Neural Network (ANN). The main two input parameters are the entrance and exit points into and from the identification region. Figure 7 shows their percentage for all five widths of cut and $a_{p}=10 \mathrm{~mm}$.

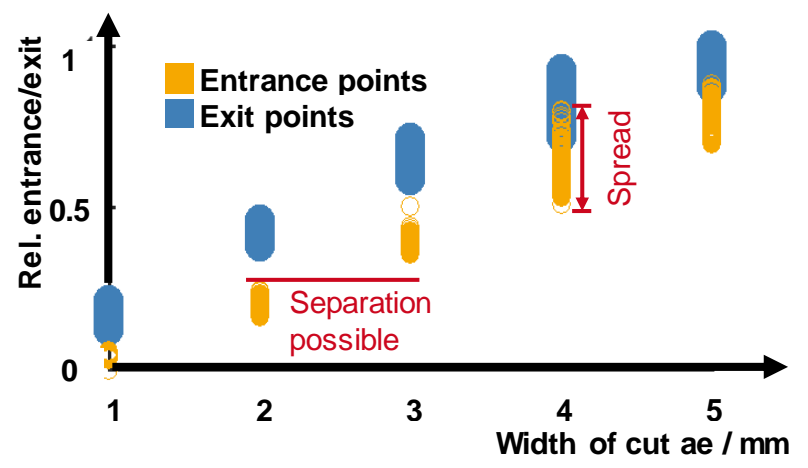

Fig. 7: Entrance and exit percentages for $a_{p}=10 \mathrm{~mm}$.

For each width of cut, 120 rotations where analyzed, each resulting into one circle for both the entrance (orange) and exit (blue) point. As expected, both the entrance and exit points for larger width of cut $a_{e}$ have a higher percentage value than for lower width of cut $a$. The ability to correctly estimate the width of cut $a_{e}$ strongly depends on how well two groups of entrance or exit points can be separated from each other, c.f. Figure 7 (red line). Another factor for the resolution of the method is the spread of a group of entrance or exit points, c.f. Figure 7 (red arrow).

Since the identification region is defined using Cartesian coordinates, the entrance and exit points also depend on the quadrant in which lies the identification region. Hence, the quadrant is the third input variable for the ANN.

As discussed earlier, the percentage of entrance or exit points from the same width of cut but different depth of cut vary. They are generally smaller for smaller depths of cut. Therefore, the depth of cut is the fourth input variable. Furthermore, it was found that using the area enclosed by a polar figure as an additional input variable increases the accuracy of the output of the ANN. Figure 8 shows the architecture of the ANN being used in this research.

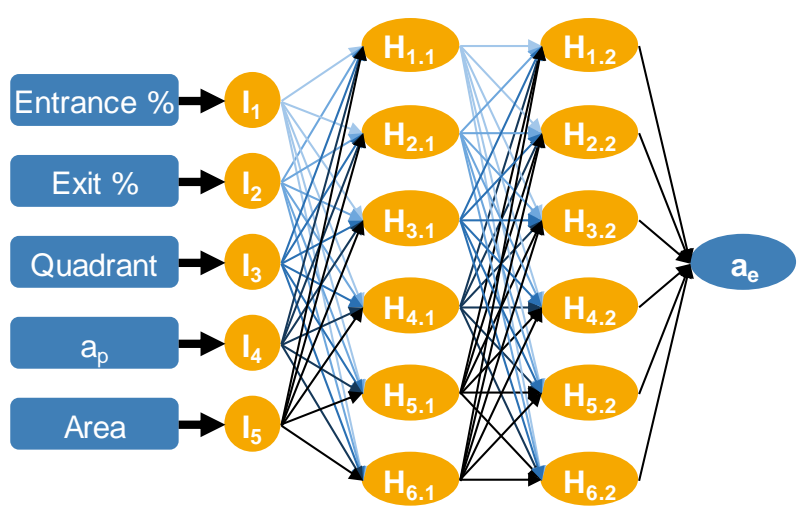

Fig. 8: Structure of the ANN.

According to TRENN [Trenn 2008], the number of hidden layers was set to 2 and the number of nodes in each hidden layer to 6 . The output of the ANN is the width of cut $a e$. The activation function is a sigmoid (logistic) function.

The training of the ANN happened using $90 \%$ of the existing 600 data sets. However, for validation, several different ANN were trained, leaving out some parameter combinations entirely to later use the ANN on them. This aspect will be shown in the following Chapter 4 .

\section{VALIDATION}

The effectiveness and accuracy of the proposed method is validated with six different models. For the first five models, polar figures of one of the five widths of cut are not considered for the training. The validation of each model was then performed using the group that was left out. For example, model 3 was trained using polar figures from $a_{e}$ values of $1,2,4$ and $5 \mathrm{~mm}$. This model was then used to predict the width of cut from polar figures of $a_{e}=3 \mathrm{~mm}$. Figure 9 shows this concept.

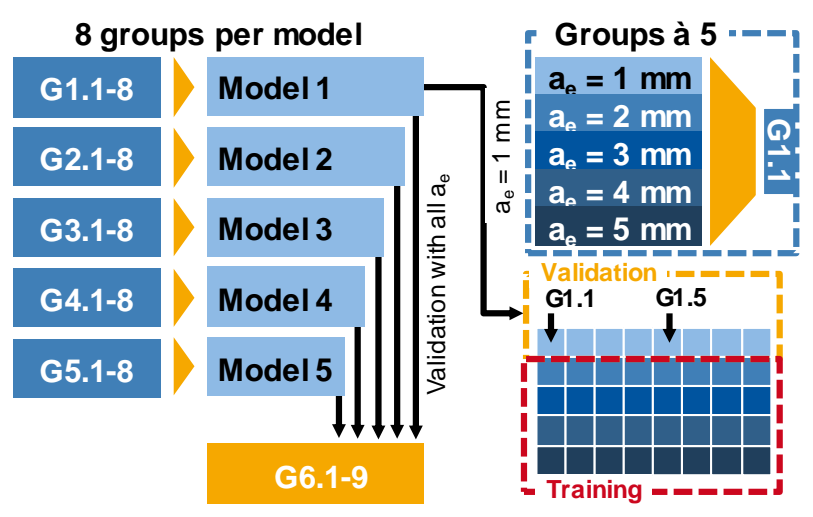

Fig. 9: Validation concept.

Each model was trained with eight groups, where each group contained data from five tool rotations per each width of cut and all three different depths of cut, resulting in 15 polar figures each. Groups 1-5 were thus trained with 480 polar figures and validated on the remaining 120 polar figures of the width of cut that was not considered for the training of that model.

A sixth model was trained, using all 600 polar figures from all widths of cut. This model 6 was then validated with nine further groups that were taken from another set of cuts with the same cutting conditions. Table 1 shows the results of the validation process. 
Tab. 1: Validation results of the error $\varepsilon$ and the standard deviation $\sigma$ of the error.

\begin{tabular}{|c|c|c|c|c|c|}
\hline & \multicolumn{2}{|c|}{ Prdct. $a_{e}=n$} & \multicolumn{2}{|c|}{ Prdct. gro } & \\
\hline & $\varepsilon$ & $\sigma$ & $\varepsilon$ & $\sigma$ & \\
\hline Model 1 & 0.590 & 0.586 & -0.701 & 1.12 & $a_{e} 1$ \\
\hline Model 2 & -0.08 & 0.051 & -0.017 & 0.085 & $a_{e} 2$ \\
\hline Model 3 & -0.04 & 0.307 & -0.015 & 0.155 & $a_{e} 3$ \\
\hline Model 4 & 0.05 & 0.120 & -0.013 & 0.093 & $a_{e} 4$ \\
\hline Model 5 & 0.08 & 0.124 & -0.029 & 0.102 & 5 \\
\hline
\end{tabular}

The quality of an estimation is determined by its accuracy and precision. The accuracy defines how close a measured or estimated value is to its true value. The precision is defined by how close repetitive measures or estimations are to each other [JCGM 2008]. In this research, accuracy is evaluated by the mean error and precision by the standard deviation of the error.

The accuracy of the models $2-5$ is very good, with the average error being at $0.0625 \mathrm{~mm}$. Their precision is also good, although worse than the accuracy $(0.1505 \mathrm{~mm})$. That means that the method determines wrongly in both directions equally, but on average the value is true. However, the standard deviation is still small compared to the magnitude of the value it shall predict, resulting in acceptable precision of the method. For model 1, both the accuracy and the precision are low. One possible reason is that for small $a_{e}$, the absolute values for the bending moment are low. This leads to a worse signal-to-noise ratio, provided the absolute values for the signal noise are constant.

The same can be seen for model 6 . It predicts the $a_{e}$ of $2-5 \mathrm{~mm}$ better than models 2-5, both in accuracy $(0.0185 \mathrm{~mm})$ and precision $(0.109 \mathrm{~mm})$. Yet, for $a_{e}=1 \mathrm{~mm}$, model 6 is even worse than model 1 . It predicts polar figures of $a_{e}=1 \mathrm{~mm}$ as $0.299 \mathrm{~mm}$ on average and does so with a standard deviation of $1.12 \mathrm{~mm}$, which is more than the difference between the individual $a_{e}$ classes.

Despite the lack of accuracy and precision for estimating small widths of cut, the validation shows that it is possible to determine the current width of cut of an end milling process from its respective polar figure. Provided the depth of cut is kept constant, this method is also able to be used for any random cutting path. However, as the method uses polar figures which contain data from an entire tool rotation, it has a limited time resolution. This time resolution is defined by the rotational speed $n$ as

$\Delta T \geq m / n$,

where $m$ is the number of rotations used for definition of the polar figure, and $\Delta T$ the time between 2 distinguishable engagement situations. There is a dilemma between a high accuracy of the method, which is defined by the virtual sample rate, and the time resolution. The virtual sample rate is increased when data of more than one tool rotation is used to define the polar figure (c.f. Chapter 2.1), i.e. when $m$ is larger than 1. A larger virtual sample rate leads to a more accurate determination of the exact entrance or leaving point, which in return should increase the accuracy and precision of the estimation method. However, increasing the virtual sample rate leads to an increase of $m$, which in return decreases the time resolution according to (1).

\section{SUMMARY AND OUTLOOK}

This research paper has shown a new method for an online estimation of the width of cut in end milling. It has introduced the concept of polar figures. The method proposed uses special features of these figures. It has been shown that the features can be used for a wide range of width of cut and are independent on the cutting speed and the feed rate. The features were identified with the help of an identification region which was defined by the sum of various polar figures. An Artificial Neural Network used these features, as well as other input parameters, to estimate the width of cut. The ANN was validated on six different models, each of them using data of a different specification than its training data. The result showed good accuracy and precision for larger widths of cut, while the smallest width of cut $\left(a_{e}=1 \mathrm{~mm}\right)$ was estimated with low accuracy and precision. Although the proposed method showed good result through most of the validation, there is still much potential for future research. While an ANN is able to provide good results, it needs to be regarded as a black box model. Future research will therefore focus on development of a more analytic approach. Using a simulation of the cutting process, it can be shown that the identification region is defined by a single tooth experiment (red, c.f. Figure 10). The respective parts of each polar figure (shades of blue) that are inside the identification region are those parts of the cutting process where only one blade is in contact (orange). For larger $a_{e}$, this situation emerges earlier (in up milling) than for smaller $a_{e}$. This explains the feasibility of the proposed method. Further research will hence use this knowledge to develop an analytical method.

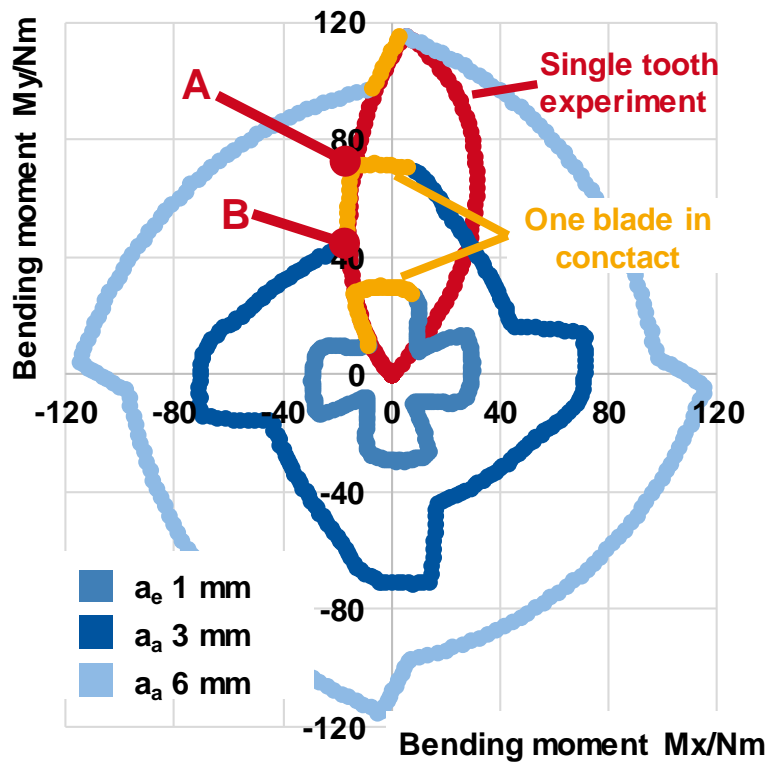

Fig. 10: Correlation between polar figures of a single tooth experiment to a 4-bladed end mill.

An industrial use of this method is to support tool wear monitoring systems, c.f. Chapter 1 . However, it is yet not understood or analyzed how tool wear effects the polar figures and hence the feasibility of the proposed method. Further research will hence also focus on this aspect.

\section{ACKNOWLEDGMENTS}

The research shown in this paper is kindly funded by the German Bundesministerium für Wirtschaft und Energie in the AiF-ZIM project "Modellentwicklung zur Berücksichtigung von Prozess-, Maschinen und 
Werkzeugeinflüssen bei der Verschleißberechnung und -prognose für spanabhebende Werkzeuge"

\section{REFERENCES}

[Teti 2010] Teti et al., "Advanced monitoring of machining operations", CIRP Annals - Manufacturing Technology 59 (2010) 717-739, 2010

[Klocke 2016] Klocke, F., Döbbeler, B., Goetz, S., "Online tool wear measurement for hobbing of highly loaded gears in Geared Turbo Fans", Procedia Manufacturing Vol 6 (2016), pp. 9-16, 2016

[Artis 2011] Artis sales Online-document (http://www.artis.de/de/files/2011/09/ARTIS_Liebherr_CT M_Waelzfraesen_de.pdf)

[Fussel 2015] Fussel et al., "Real-time tool wear monitoring in milling using a cutting condition independent method", International Journal of Machine Tools \& Manufacture 89 (2015), pp. 1-13, 2015

[Salgado 2013] Salgado et al., "Tool wear estimation for different workpiece materials using the same monitoring system", Procedia Engineering 63 (2013), pp. 608 - 615, 2013

[Nouri 2015] Nouri, M. et al. "Real-time tool wear monitoring in milling using a cutting condition independent method", International Journal of Machine Tools and Manufacture 89 (2015), pp. 1-13

[Emec 2016] Emec, S., Krueger, J., Seliger, G., "Online fault-monitoring in machine tools based on energy consumption analysis and non-invasive data acquisition for improved resource-efficiency", Procedia CIRP 40 (2016), pp. $236-243,2016$

[Jáuregi 2018] Jáuregi, J. et al., "Frequency and TimeFrequency Analysis of Cutting Force and Vibration Signals for Tool Condition Monitoring", IEEE access volume 6 (2018), pp. 6400-6410,

DOI 10.1109/ACCESS.2018.2797003

[Trenn 2008] Trenn, S. "Multilayer perceptrons: approximation order and necessary number of hidden units" IEEE Transactions on Neural Networks, vol. 19, no. 5 (2018), pp. 836-844, 2008.

[JCGM 2008] JCGM200:2008, International vocabulary of metrology - Basic and general concepts and associated terms (VIM)

https://www.bipm.org/utils/common/documents/jcgm/JCG M_200_2008.pdf 